\title{
Simultaneous Cervical and Lumbar Spine Surgery: Retrospective Analysis of 45 Cases
}

\author{
Amit Babasaheb Aiwale ${ }^{1} \quad$ Pankajkumar Ranchhodbhai Patel ${ }^{1} \quad$ Syed Ameer Basha Paspala ${ }^{1}$ \\ T.V. Ramakrishna Murthy ${ }^{1}$
}

${ }^{1}$ Department of Neurosurgery, Care Hospital, Hyderabad,

Telangana, India

Address for correspondence Amit Babasaheb Aiwale, MS/DNB Neurosurgery, Department of Neurosurgery, Care Hospital, Road No. 1, Banjara Hills, Hyderabad 500034, Telangana, India (e-mail: aiwale_amit@yahoo.com).

Indian J Neurosurg 2019;8:47-52

\begin{abstract}
Keywords

- tandem spinal stenosis

- simultaneous cervicolumbar

- spine surgery

Background The term 'tandem spinal stenosis' (TSS) was first introduced by Dagi et al to describe concurrent symptomatic cervical and lumbar spinal stenosis. A typical clinical picture includes intermittent neurogenic claudication, myelopathy, and polyradiculopathy in both the upper and lower extremities. The incidence of TSS ranges from 0.12 to $28 \%$.

Methods We studied patients who presented with tandem canal stenosis and operated cervicolumbar decompression with or without fusion procedures by two separate neurosurgical teams simultaneously from June 2015 to 2017 with follow-up period of minimum 6 months.

Results We had 30 (66.66\%) male and 15 (33.33\%) female patients who underwent simultaneous cervical and lumbar spine surgeries. The average age was 57.8 years (male) and 53.9 years (female). Cervical canal stenosis was graded as per magnetic resonance imaging (MRI) morphological grades of stenosis by Kang et al and lumbar grading, was done as per Schizas et al grading system. The mean duration of complaints in cervical and lumbar compression was $29.54 \pm 44.99$ months and $30.55 \pm 38.11$ months, respectively. The mean preoperative Japanese Orthopaedic Association (JOA) score of was $10.46 \pm 1.39$, whereas the postoperative mean JOA score was $11.93 \pm 1.28$, and mean preoperative $(38.59 \pm 16.52)$ and postoperative $(29.22 \pm 9.38)$ Oswestry Disability Index (ODI) scores showed a statistically significant difference $(p=0.0001)$. Conclusion Patients with TSS are elderly and have associated comorbidities, still simultaneous cervical and lumbar surgery is feasible with the good outcome if you have two neurosurgical teams operating simultaneously and having good other super specialty teams' support. It can be timesaving and cost effective for patients. Also, it avoids patients from undergoing exposure to two separate surgical and anesthetic stress.
\end{abstract}

\section{Introduction}

The term 'tandem spinal stenosis' (TSS) was first introduced by Dagi et al. ${ }^{1}$ to describe concurrent symptomatic cervical and lumbar spinal stenosis. A typical clinical picture includes intermittent neurogenic claudication, progressive gait disturbance, and a combination of myelopathy and polyradiculopathy in both the upper and lower extremities. The incidence of TSS has been reported, which ranges from 0.12 to $28 \%{ }^{2}$ The process of spondylotic degeneration that gives rise to lumbar stenosis might be responsible for tandem cervicolumbar stenosis.

Tandem spinal stenosis being uncommon, there is still a controversy in the surgical strategy of these patients. Staged surgery (cervical followed by lumbar or vice versa) or simultaneous surgery has been advocated. ${ }^{3,4}$ Although not statistically determined, most would choose decompression of one region in accordance with each patient's predominant clinical symptoms and regard one-staged decompression received

July 6, 2018

accepted

July 30, 2018

published online

March 27, 2019
DOI https://doi.org/

10.1055/s-0039-1677962

ISSN 2277-954X.
C2019 Neurological Surgeons'

Society of India
License terms

(이 (1) $\Theta \circledast$ 
as very invasive in this elderly group. In this retrospective study, we analyze patients who presented with tandem cervicolumbar spinal stenosis and underwent simultaneous surgery for both cervical and lumbar decompression with or without fusion procedures by two neurosurgical teams.

\section{Materials and Methods}

Patients who presented with tandem cervicolumbar canal stenosis and underwent simultaneous single-stage surgery involving cervicolumbar decompression with or without fusion procedures from June 2015 to 2017 with follow-up period of minimum 6 months. All patients were diagnosed based on clinical presentation, examination, and magnetic resonance imaging (MRI) findings. The radiological severity of cervical and lumbar stenosis was analyzed with quantitative grading systems. - Fig. 1 represents the MRI sigital image of two patients included in the study. Morphological grades of stenosis for cervical by Kang et $\mathrm{al}^{5}$ and lumbar by Schizas et $\mathrm{al}^{6}$ were used, respectively. All patients had preanesthetic evaluations and fitness clearance from super specialists, such as cardiologist, endocrinologist, and pulmonologist as required.

The surgery involved multiple surgeons from neurosurgery team divided into two teams operating simultaneously at cervical and lumbar levels to reduce the operative time and

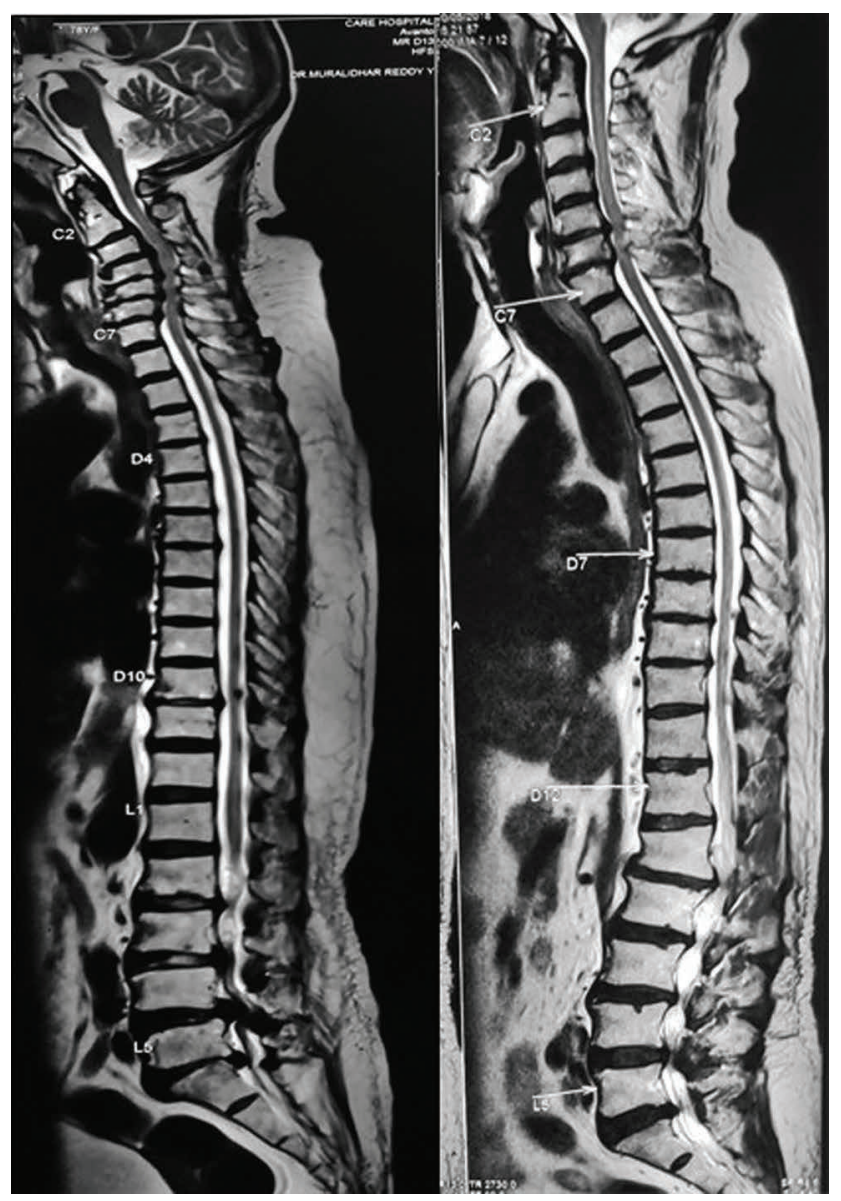

Fig. 1 MRI T2 sagittal images of two patients of tandem spinal cervicolumbar stenosis. MRI, magnetic resonance imaging. intern long exposure to anesthetic drugs. All patient received single-dose methylprednisolone injection preoperatively. Patient's demographics, surgical blood loss, blood transfusion required, total intensive care unit (ICU) and hospital stay, perioperative complications, the requirement of the drain, and drain output were recorded. The clinical results were evaluated according to the Nurick's grade $(1972)^{7}$ and the modified Japanese Orthopaedic Association (mJOA) score $^{7}$ for cervical myelopathy and Oswestry Disability Index (ODI) score for low back. ${ }^{8}$ All patients received training from a physiotherapist and were advised to continue post discharge. Data were recorded and analyzed by a statistician with an appropriate statistical test as required.

\section{Results}

In this study, we had 30 (66.66\%) male and 15 (33.33\%) female patients who underwent simultaneous cervical and lumbar spine surgeries. The average age of male and female patients included in this study was $57.8 \pm 9.46$ years and $53.9 \pm 11.48$ years, respectively. Out of all 45 patients, 1 patient had single-level cervical pathology, 2 patients had two-level pathology, while 42 (i.e., 93.4\%) patients had threeor more cervical level pathologies. While 22 (48.9\%) patients had single-level lumbar pathology, 19 (42.2\%) patients had the double-level, and only 1 (2.2\%) patient had triple-level lumbar pathology. - Fig. 2 represents the postoperative X-ray of a patient who underwent simultaneous cervical and lumbar spine fixation. Cervical canal stenosis was graded as per morphological grades of stenosis for cervical by Kang et $\mathrm{al}^{5}{ }^{5}$ which involved 1 (2.22\%) patient with grade 1,24 (53.33\%) patients with grade 2 , and 20 (44.44\%) patients with grade 3 cervical stenosis. For lumbar canal stenosis, MRIbased morphological grading was done as per by Schizas et $\mathrm{al}^{6}$ grading system. Of which, 11 (24.44\%) patients had grade B, $20(44.44 \%)$ patients had grade C, and 14 (31.11\%) patients had grade $\mathrm{D}$ lumbar canal stenosis. The mean duration of complaints in cervical and lumbar compression was $29.54 \pm 44.99$ months and $30.55 \pm 38.11$ months, respectively.

Patients were analyzed by JOA score, Nurick grade, and ODI score. The mean preoperative JOA score of the patients was $10.46 \pm 1.39$, whereas the postoperative mean JOA score

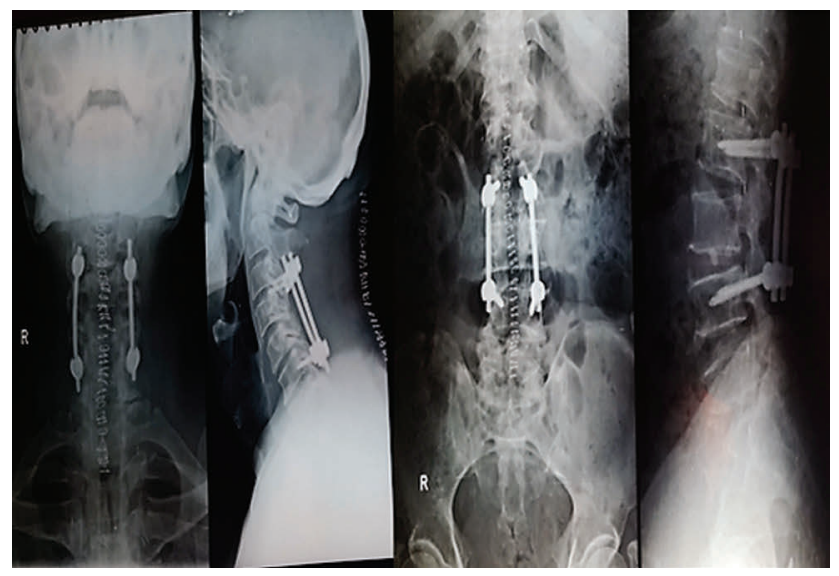

Fig. 2 Postoperative images cervical and lumbar spine X-ray. 
was $11.93 \pm 1.28$ and showed the statistically significant difference $(p=0.0001)$. A similar trend was observed with respect to mean preoperative $(38.59 \pm 16.52)$ and postoperative $(29.22 \pm 9.38)$ ODI scores $(p<0.01)$. In this study, 41 patients had the cervical fixation with implants, while 33 patients had lumbar fixation done with implants. The mean operative time was $108.88 \pm 21.12$ minutes. The average blood loss was $474.44 \pm 162.32 \mathrm{~mL}$ during the procedure. Only two patients required postoperative packed red blood cells (PRBC) transfusion to stabilize patient hemodynamically. The comparison and correlation analysis of different factors with respect to preoperative and postoperative analysis have been shown in - Table $\mathbf{1}$.

In this study, we had two patients who developed postoperative surgical site infection, which was managed with repeated dressing and appropriate antibiotic. One patient required postoperative ventilation support for 1-day and 3-day ICU stay for adequate respiratory recovery. One patient who had intraoperative dural tear was managed with placement of dural substitute to minimize operative time. Postoperative drain placement was avoided as far as possible unless strongly indicated.

\section{Discussion}

Teng and Papatheodorou ${ }^{9}$ in 1964 first described the phenomenon of concurrent cervical and lumbar stenosis, coining the term "tandem stenosis." Tandem stenosis has a reported prevalence ranging from 5 to 25\% in different series. There has been a lot of controversies about management of these patients. There has been a debate about whether the most symptomatic level should be operated first, and then the less symptomatic or upper level should be operated followed by the lower symptomatic level. Although Benini ${ }^{10}$ reported that a simultaneous decompression in such cases is impossible, Dagi et $\mathrm{al}^{1}$ were the first to report the simultaneous decompression of the cervical and lumbar spines in cases of tandem stenosis. Their group determined the sequencing of surgery based on the level that was most clinically symptomatic or, if areas appeared equally symptomatic, based on the degree of stenosis by myelography. In instances of the equivalent severity of symptoms and degree of stenosis, a simultaneous decompressive procedure was performed. But no analysis was done to determine whether the outcomes were appreciably different in this subset of patients compared with patients who underwent sequential decompression on two separate occasions. Few authors also claim to have a higher risk of cervical injury in these patients if they are first planned for lumbar decompression and then go for cervical decompression.

Eskander et $\mathrm{al}^{11}$ in their study concluded that patients with TSS can be effectively managed with either simultaneous or staged procedure, but plan should be tailored according to age, and options should be used to minimize the blood loss and operative time. In our study, every effort was taken to minimize the blood loss, and as two neurosurgical teams operated simultaneously at different levels, the total operative time was effectively minimized. Naderi and Mertol ${ }^{12}$ have reported the case and concluded that simultaneous surgery for different segments of the spine is an alternative approach in patients with combined symptomatic pathologies, whose general or social condition is risky for two long-lasting procedures. Authors also suggested tapering the surgical procedure as per patient's clinical parameters to minimize surgical stress and operative time. When we compared results of our study with the past studies, results were comparable as shown in - Table 2.

When postoperative ODI scores and Nurick grades were compared in patients below 60 years and above 60 years, these were found to be statistically significant $(p \leq 0.01)$, suggesting age is an important factor in determining a postoperative outcome. Also, postoperative JOA scores in patients with $<400$ or $>400 \mathrm{~mL}$ blood loss had a significant association. These findings were in correlation with the literature. Krishnan et al in their study had similar findings as age and blood loss had the significant impact on outcome in patients managed with simultaneous cervicolumbar surgeries. ${ }^{13}$

Change in ODI score and Nurick grade in patients with more than two vertebral levels was significantly less compared with that in patients who were operated for less than two levels. Similar findings were noted in postoperative ODI scores in lumbar surgeries when less than two and more than two vertebral levels. However, for the obvious reason that patient with less vertebral level pathologies will require less dissection and operative time will have lesser blood loss, there is a higher chance of better postoperative scores and better prognosis.

When patients were compared based on whether fixation was done or not, all scores, i.e., mJOA, ODI score, and Nurick grade, were found to have no association, and no statistical significance was found suggesting that fixation with implant had no adverse outcome in prognosis. However, interestingly, patients with cervical implants had the higher change in ODI scores compared with ODI scores in those who did not have cervical implant fusion, while a patient with lumbar implants had lower mJOA score compared with that in those who did not have lumbar implant fixation. Yehya in his study "The clinical outcome of lateral mass fixation after decompressive laminectomy in cervical spondylotic myelopathy" got similar findings as lateral mass fixation has better postoperative clinical outcomes when compared with only cervical laminectomy. ${ }^{14}$

Postoperative mJOA and Nurick grade were statistically significant when the operative time was divided into $<120$ minutes and > 120 minutes, suggesting the operating time has an impact on postoperative recovery. When preoperative and postoperative mJOA, Nurick, and ODI scores were analyzed in this study, there was an improvement in these scores, suggesting the better outcome of the simultaneous surgery at cervical and lumbar level. However, these scores are at 6 months follow-up, and longer follow-up of these patients is required for comparison with other studies show in $\mathbf{- F i g} \mathbf{3}$.

As cervical and lumbar are two different levels, the specific arrangement of surgical teams, nursing staff, instrument trollies, C-arm, and anesthetic support can be done as shown in -Fig. 4 for hassle-free surgery. If the surgery is cervical with dorsal or dorsal with lumbar level, simultaneous 


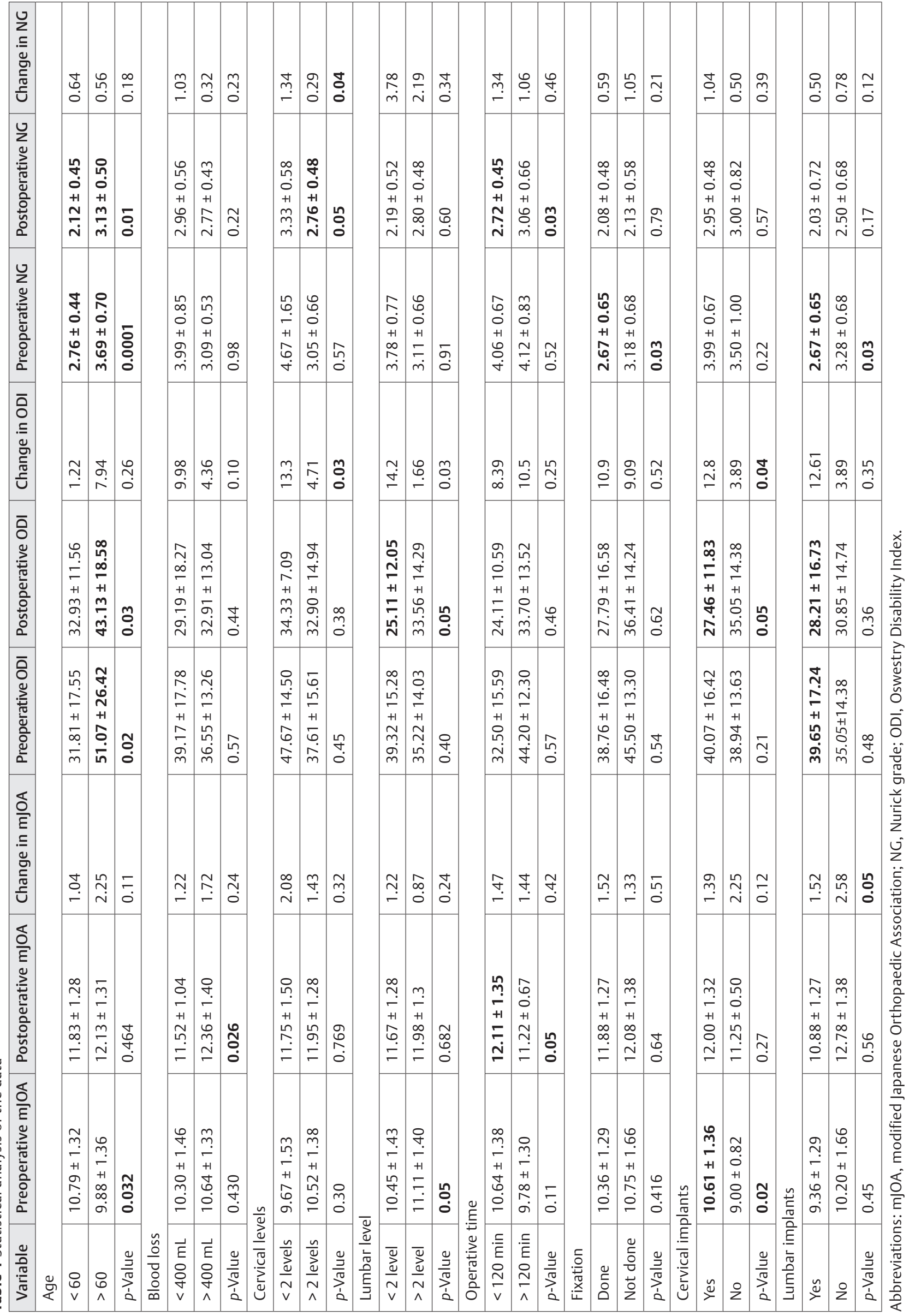


Table 2 Comparison with previous studies

\begin{tabular}{|l|l|l|l|l|l|l|l|}
\hline & No of patients & \multicolumn{2}{|c|}{ mJOA score } & \multicolumn{2}{c|}{ Nurick grade } & \multicolumn{2}{c|}{ ODI score } \\
\hline & & Preoperative & Postoperative & Preoperative & Postoperative & Preoperative & Postoperative \\
\hline $\begin{array}{l}\text { This } \\
\text { study }\end{array}$ & 45 & $10.46 \pm 1.39$ & $11.93 \pm 1.28$ & $3.08 \pm 0.7$ & $2.86 \pm 0.5$ & $38.59 \pm 16.52$ & $29.22 \pm 9.38$ \\
\hline $\begin{array}{l}\text { Kikuike } \\
\text { et al }\end{array}$ & 17 & $14.4 \pm 6.1$ & $14.8 \pm 9.5$ & NA & NA & NA & NA \\
\hline $\begin{array}{l}\text { Eskander } \\
\text { et al }{ }^{11}\end{array}$ & 21 & 10.14 & 12.45 & & & 60.64 & 23.18 \\
\hline $\begin{array}{l}\text { Krishnan } \\
\text { et al }\end{array}$ & 53 & 8.8 & $14.48 \pm 1.89$ & 3.831 & 1.96 & 68.15 & 24.93 \\
\hline
\end{tabular}

Abbreviations: mJOA, modified Japanese Orthopaedic Association; NA, not applicable; ODI, Oswestry Disability Index.
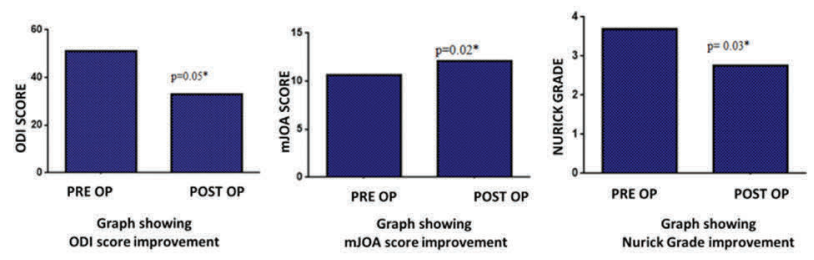

Fig. 3 Pre-and postoperative clinical grading comparison. mJOA, modified Japanese Orthopaedic Association; NG, Nurick grade' ODI, Oswestry Disability Index.

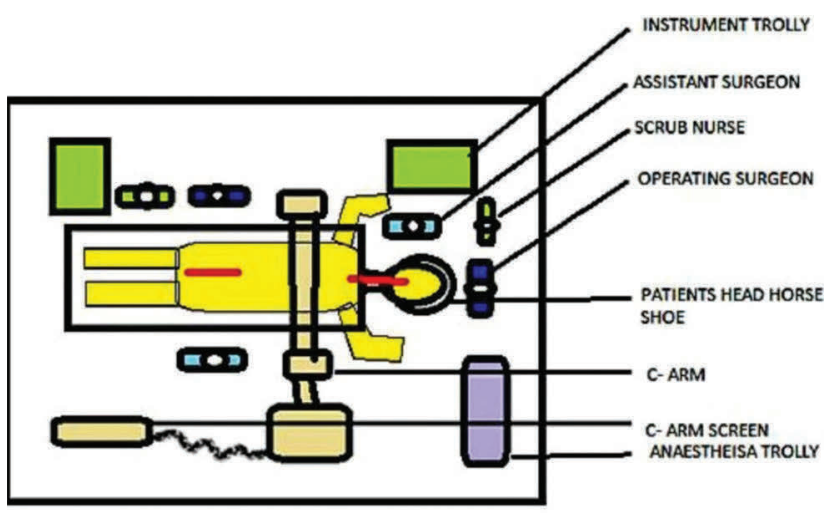

Fig. 4 OT setup arrangement for two surgical teams for simultaneous cervicolumbar surgery. OT, operation theatre.

surgery would not be possible due to inadequate space for the arrangement.

\section{Limitations}

This is a retrospective study with a follow-up period of 6 months. It would be early to predict the long-term outcomes. This is a single-center study done by two experienced neurosurgery teams, and outcomes in other centers need to be evaluated for uniformity. This is not comparative study to suggest stepwise spinal decompression is better or simultaneous decompression.

\section{Conclusion}

Though TSS occurs relatively infrequently, the unrecognized occurrence in the general population may be higher. Detailed examination for even subtle signs followed by whole spine MRI (T2 sagittal) screening should be done. Even though these patients are elderly and have associated comorbidities, simultaneous cervical and lumbar surgery is feasible with the good outcome if you have two neurosurgical teams operating simultaneously and having good other super specialty team's support. It can be timesaving and cost effective for patients. Also, it avoids patients from undergoing exposure to two separate surgical and anesthetic stress.

\section{Conflict of Interest}

There are no conflicts of interest related to this study.

\section{References}

1 Dagi TF, Tarkington MA, Leech JJ. Tandem lumbar and cervical spinal stenosis. Natural history, prognostic indices, and results after surgical decompression. J Neurosurg 1987;66(6):842-849

2 Epstein NE, Epstein JA, Carras R, Murthy VS, Hyman RA. Coexisting cervical and lumbar spinal stenosis: diagnosis and management. Neurosurgery 1984;15(4):489-496

3 Aydogan M, Ozturk C, Mirzanli C, Karatoprak O, Tezer M, Hamzaoglu A. Treatment approach in tandem (concurrent) cervical and lumbar spinal stenosis. Acta Orthop Belg 2007;73(2):234-237

4 Kikuike K, Miyamoto K, Hosoe H, Shimizu K. One-staged combined cervical and lumbar decompression for patients with tandem spinal stenosis on cervical and lumbar spine: analyses of clinical outcomes with minimum 3 years follow-up. J Spinal Disord Tech 2009;22(8):593-601

5 Kang Y, Lee JW, Koh YH, et al. New MRI grading system for the cervical canal stenosis. AJR Am J Roentgenol 2011;197(1):W134-40

6 Schizas C, Theumann N, Burn A, et al. Qualitative grading of severity of lumbar spinal stenosis based on the morphology of the dural sac on magnetic resonance images. Spine 2010;35(21):1919-1924

7 Williams KE, Paul R, Dewan Y. Functional outcome of corpectomy in cervical spondylotic myelopathy. Indian J Orthop 2009;43(2):205-209

8 Fairbank JCT, Pynsent PB. The Oswestry disability index. Spine 2000;25(22):2940-2952, discussion 2952 
9 Teng P, Papatheodorou C. Combined cervical and lumbar spondylosis. Arch Neurol 1964;10:298-307

10 Benini A. Zervikale und lumbale Stenose. In: Benini A, ed. Ischias ohne Bandscheibenvorfall: Die Stenose des lumbalen Wirbelkanal. Bern: Verlag Hans Huber, 1986:84

11 Eskander MS, Aubin ME, Drew JM, et al. Is there a difference between simultaneous or staged decompressions for combined cervical and lumbar stenosis? J Spinal Disord Tech 2011;24(6):409-413

12 Naderi S, Mertol T. Simultaneous cervical and lumbar surgery for combined symptomatic cervical and lumbar spinal stenoses. J Spinal Disord Tech 2002;15(3):229-231, discussion 231-232

13 Krishnan A, Dave BR, Kambar AK, Ram H. Coexisting lumbar and cervical stenosis (tandem spinal stenosis): an infrequent presentation. Retrospective analysis of single-stage surgery (53 cases) Eur Spine J 2014;23(1):64-73

14 Yehya $A$. The clinical outcome of lateral mass fixation after decompressive laminectomy in cervical spondylotic myelopathy. Alexandria J Med. 2015;51(2):153-159 